\title{
Étude expérimentale de la remise en suspension des particules
}

\section{Comparaison entre revêtement lisse et rugueux}

\author{
J. El Hijri ${ }^{1,2, a}$, A. Draoui ${ }^{1}$ et K. Limam ${ }^{2}$ \\ 1 Laboratoire d'Energétique, Équipe des Transferts Thermiques et Energétique (ETTE), FST, BP 416, Tanger, Maroc \\ 2 LEPTAB, Pôle Sciences et Technologie, Av. Michel Crépeau, 17042 La Rochelle Cedex 1, France
}

\begin{abstract}
Résumé - Concernant la pollution particulaire des ambiances, qu'elles soient habitables ou destinées à accueillir une population parfois importante (telles que les grands halls publics, les gares ou lieux de vie), c'est essentiellement l'étude du dépôt des particules ou de leur migration dans l'air, qui a le plus souvent été traité dans la bibliographie. Pour l'instant trop peu d'études ont été consacrées aux aspects de la remise en suspension des particules car de façon générale, qu'il s'agisse d'habitations ou de grands volumes ouverts au public, les vitesses de l'air restent relativement faibles et permettent difficilement d'« arracher » les particules déposées. C'est dans cet esprit que nous avons élaboré en cellule-test, un protocole expérimental pour mettre en évidence ce phénomène. Nos expérimentations permettront une quantification de la contribution de l'activité humaine (déplacement par la marche) dans la remise en suspension de particules initialement sur le plancher. Le paramètre sur lequel nous nous sommes focalisés ici concerne la rugosité du revêtement posé au plancher (même nature mais de rugosité différente). Nos résultats permettront d'une part de caractériser le facteur humain comme une «pseudo-source » de pollution particulaire, et d'autre part, de l'inclure dans la caractérisation particulaire des ambiances.
\end{abstract}

Mots clés : Pollution / particules / dépôt / remise en suspension / revêtement du plancher

Abstract - Particles pollution inside buildings which are designated for living or to welcome peoples (big number of persons in buildings such as big public halls or in underground stations) is a very important subject treated in bibliography for its parameters such as particles settling and airborne transport. Nowadays there is a lack of studies developed in the frame of particles suspension in air, as the air speed in big volume rooms is low and so more difficult take off the settled particles. Therefore we have developed a testing room to measure this phenomenon. Our experiments showed out the relation between the settled particles quantity that have been arisen by the human movements inside the testing room as a function of the latest and also the inside air quality as a function of the number of persons and their activities. The parameters which could be varied were: the number of persons inside the testing room and the floor materials. Our experiments took into consideration the human activities as a source of air pollution and also as a factor which influences the inside air quality.

Key words: Pollution / particle / deposition / resuspension / floor materials

\section{Introduction}

L'environnement intérieur d'un bâtiment constitue un milieu complexe caractérisé par de nombreux paramètres qui peuvent avoir un impact sur la santé et le confort des occupants. Il existe de multiples interactions entre le bâtiment, son utilisation et les personnes qui y vivent ou y travaillent. C'est aussi un environnement dynamique caractérisé par une grande variabilité des sources de pollution, par des types d'espaces intérieurs différents, et par

\footnotetext{
a Auteur pour correspondance:
}

jelhij02@univ-lr.fr

\section{Nomenclature}

\begin{tabular}{|ll|}
\hline$C_{\mathrm{i}}$ & $\begin{array}{l}\text { concentration intérieure en particules dans l'air } \\
\end{array}$ \\
$C_{\text {ext }}$ & concentration extérieure en particules dans l'air \\
$C_{\mathrm{Di}}$ & concentration en particules déposées \\
$S_{\mathrm{i}}$ & source interne de particules $\left(\mathrm{kg}\right.$ ou nombre $\left.\mathrm{m}^{-3} \cdot \mathrm{s}^{-1}\right)$ \\
$\lambda_{\mathrm{r}}$ & coefficient de renouvellement d'air $\left(\mathrm{s}^{-1}\right)$ \\
$f$ & coefficient de pénétration \\
$\lambda_{\mathrm{de}}$ & paramètre de dépôt $\left(\mathrm{s}^{-1}\right)$ \\
$\lambda_{\mathrm{R}}$ & paramètre de remise en suspension $\left(\mathrm{s}^{-1}\right)$ \\
\hline &
\end{tabular}


des conditions de «climat intérieur » et de ventilation également diverses.

Les principales sources de contaminations sont les occupants eux-mêmes et leurs activités, les matériaux de construction (revêtement des murs, peintures, matériaux d'isolation...), ainsi que les équipements du bâtiment (appareils ménagers, systèmes de ventilation et d'air conditionné... ).

La qualité de l'air extérieure a fait l'objet de beaucoup d'études mais ce n'est que plus récemment, que la communauté scientifique a commencé à s'intéresser à la pollution dans les environnements fermés.

De nombreux travaux de recherches ont démarré dans le but d'étudier les particules et leur dépôt sur les parois verticales, sol et plafonds [1-3]. Des études sur la remise en suspension des particules radioactives, des sédiments sous marins, etc. font également l'objet de certaines recherches approfondies. Or, l'étude de la remise en suspension des particules dans l'habitat par les pas ou autre cause, reste un domaine encore très peu exploré, ce qui a engendré une connaissance partielle, voire inexistante de certains scénarios sur le phénomène.

Il faut savoir que les causes de remise en suspension de particules dans le bâtiment sont nombreuses; les cycles arrêt-marche des ventilations mécaniques contrôlées (VMC) conduisent à remettre en suspension les particules déposées dans les gaines [4], même si les gammes de vitesse de soufflage restent insuffisantes pour arracher les particules déposées sur les parois intérieures (murs, plancher et plafond) de la pièce ventilée. Le déplacement des personnes à l'intérieur provoque aussi une remise en suspension par les semelles et par les perturbations locales de l'écoulement de l'air [5].

\section{Comportement des particules dans les ambiances intérieures}

Pour déterminer la qualité particulaire de l'air intérieur, il faut considérer les différents échanges qui entrent en jeu. La vie d'une particule, en tant que quantité polluante de l'air intérieur, est constituée de trois étapes. La première est son transport dans l'air sous l'action locale des écoulements de l'air et des différentes forces qui peuvent s'exercer sur elle (diffusion moléculaire, gravité, forces radiométriques). La deuxième étape est son interaction avec une surface, soit la particule sera captée par la surface (et donc sera perdue pour l'air) soit elle ricochera, ce résultat dépendra de la nature et de la force de l'interaction qui s'opère entre la surface et la particule. La dernière étape est, éventuellement, la remise en suspension de ladite particule par un phénomène tierce (courant d'air, activité humaine. . .) $[4,5]$.

Le bilan de conservation massique du polluant particulaire dans une enceinte mono zone [6], peut être exprimé par la relation suivante:

$$
\frac{\mathrm{d} C_{\mathrm{i}}}{\mathrm{d} t}=f \times \lambda_{\mathrm{r}} C_{\mathrm{ext}}-\lambda_{\mathrm{r}} C_{\mathrm{i}}-\lambda_{\mathrm{de}} C_{\mathrm{i}}+\lambda_{\mathrm{R}} C_{\mathrm{Di}}+S_{\mathrm{i}}
$$

Les deux premiers termes de cette équation correspondent aux échanges entre l'intérieur et l'extérieur. L'air pollué extérieur pénètre dans la zone par le système de ventilation ou par les différentes ouvertures, et une partie de l'air intérieur sort de cette zone par extraction. Les deux termes suivants modélisent l'interaction avec les parois (dépôt et remise en suspension). Le dernier terme correspond à la présence d'une source de particules à l'intérieur de la zone d'étude.

C'est exactement la quantification de ce terme source qui est au centre de notre travail. La présence d'être humain dans un espace «pollué » et fermé, pourrait être assimilée à une pseudo-source de pollution particulaire, l'activité humaine remettant en suspension les particules déjà déposées sur les parois.

C'est dans cet esprit que nous avons élaboré en cellule test, un protocole expérimental pour mettre en évidence le phénomène de remise en suspension par les pas d'une personne qui marche sur un plancher recouvert d'un revêtement que l'on a commencé par caractériser. Le protocole a permis de tester la capacité du revêtement à se décharger par l'action mécanique (marche) du polluant qui le recouvre. Le polluant utilisé pour générer l'aérosol est de la poudre d'alumine poly dispersée autours d'un diamètre de $0,3 \mu \mathrm{m}$.

\section{Protocole expérimental}

Nos expériences se sont déroulées dans la cellule test du LEPTAB $\left(2,5 \times 1,5 \times 2,5 \mathrm{~m}^{3}\right)$ avec une installation de VMC à débit réglable et un soufflage d'air comprimé filtré (voir Figs. 1a et b).

Le revêtement est installé sur le plancher de la cellule test, puis les compteurs de particules Grimm ${ }^{\circledR}$ (modèle 1.108) selon la disposition montrée dans la figure cidessous (deux au sol et un surélevé). Les particules sont injectées dans la cellule à l'aide d'un générateur d'aérosol TOPAS $^{\circledR}$ SAG 410 , et neutralisées de leurs charges électrostatiques par un neutraliseur TOPAS ${ }^{\circledR}$ EAN 581. L'homogénéisation de la pollution se fait par brassage, assuré par un ventilateur plafonnier.

En plus de la VMC, le soufflage direct d'air comprimé filtré à l'intérieur de la cellule contribue au nettoyage de l'air intérieur et oblige les particules à se déposer sur le plancher et les parois.

Une fois les particules déposées, on arrête la VMC et l'air comprimé pour permettre à un témoin humain de marcher dans la cellule pendant une durée de $5 \mathrm{~min}$, selon un marquage et une cadence déterminés.

La possibilité d'utiliser plusieurs types de revêtements (linoléum lisse et rugueux pour notre étude) nous permet de mieux caractériser les paramètres influençant la remise en suspension, et constitue un complément aux différentes études déjà menées sur le dépôt particulaire à l'intérieur des bâtiments.

Une analyse de surface des revêtements (rugosité et dureté) a été pratiquée préalablement pour pouvoir caractériser les matériaux et de disposer de ces données lors de la phase de modélisation. 


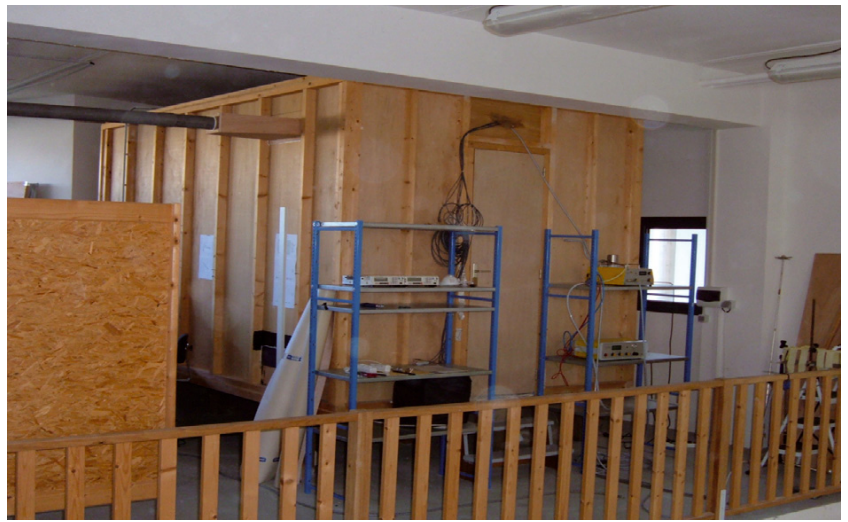

(a)

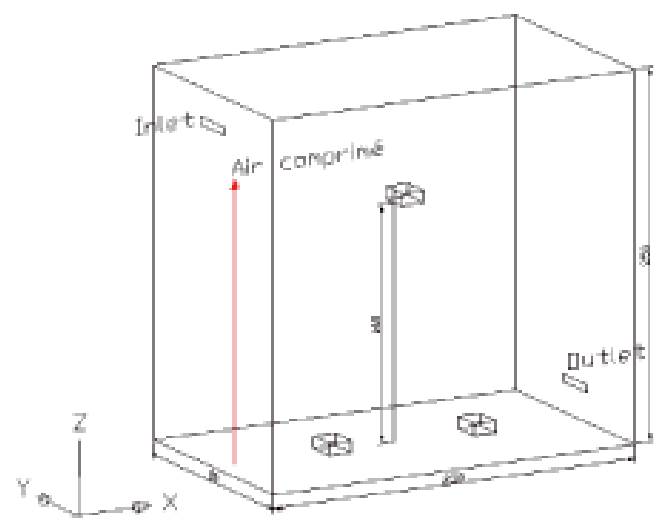

(b)

Fig. 1. a) Cellule test du LEPTAB. b) Schéma du protocole expérimental (emplacement des grimms, injection de l'air comprimé, inlet et outlet).

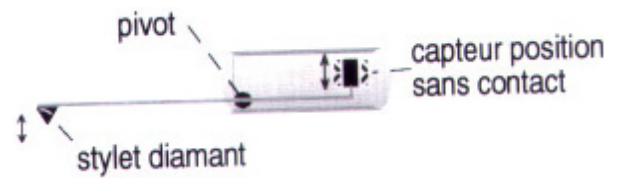

Fig. 2. Stylet pivotant.

\section{Résultats des mesures}

On distingue plusieurs méthodes d'analyses plus ou moins précises et plus ou moins adaptées au type de revêtement à étudier.

Pour nos mesures, on a utilisé un capteur mécanique composé d'un stylet pivotant muni à une extrémité d'une pointe diamant reposant sur la surface à analyser et d'un système de mesure de déplacement du bras.

La mesure du déplacement utilise le plus souvent un principe inductif. Un noyau ferrite solidaire du stylet se déplace dans une bobine à deux enroulements, le conditionneur du capteur effectuant l'excitation de la première bobine et la détection synchrone du signal induit sur la deuxième bobine. On représente ainsi les différences de bosses et de creux le long d'une ligne.

Le matériel utilisé est un MMB (Microscope Mécanique à Balayage). C'est un outil d'observation et

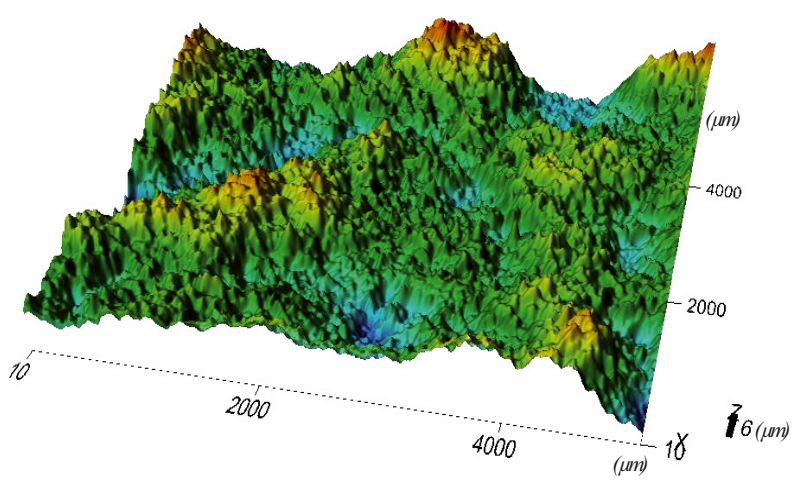

Fig. 3. Linoléum lisse.

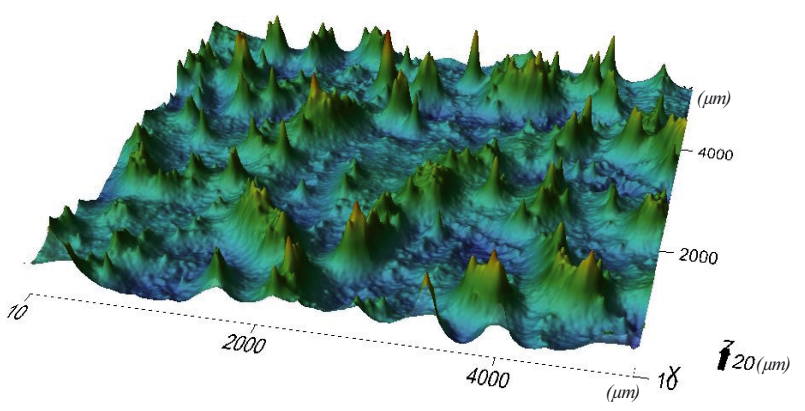

Fig. 4. Linoléum antidérapant (rugueux).

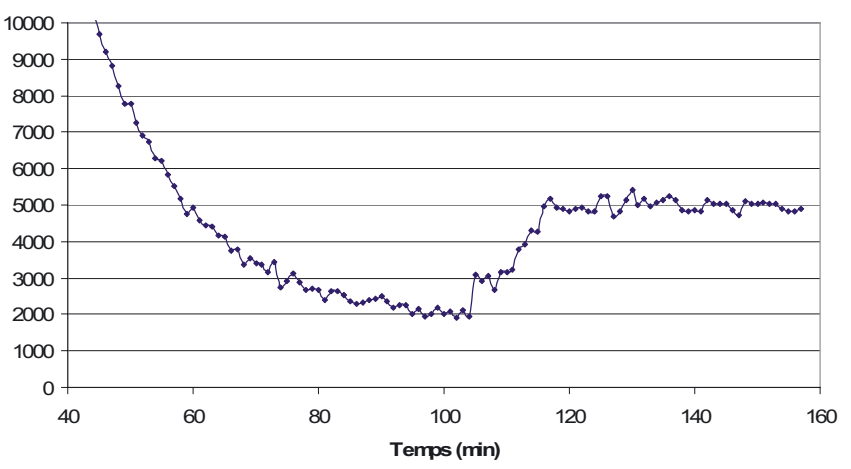

Fig. 5. Évolution du nombre de particule de $0,3 \mu \mathrm{m}$ en fonction du temps (linoléum lisse).

de caractérisation des états de surface. Il mesure les variations d'altitude de la surface des matériaux et quantifie la topographie à l'aide d'outils issus de la statistique, du traitement de signal ou du traitement d'image.

Les résultats de l'analyse de surface ont permis l'élaboration des cartographies 3D (Figs. 3 et 4) [6].

Une mesure de dureté des deux revêtements choisis a été effectuée par la société AKILOG ${ }^{\circledR}$ [7].

Les duretés des deux revêtements sont énoncées ci-après :

- Linoléum lisse : 79,44 (écart-type : 0,51);

- Linoléum rugueux : 60,96 (écart-type : 1,56).

Les résultats relatifs aux concentrations de particules sont montrés dans les figures 5 et 6 .

Le procédé de nettoyage appliqué (air comprimé propre + VMC) permet de diminuer le nombre de 


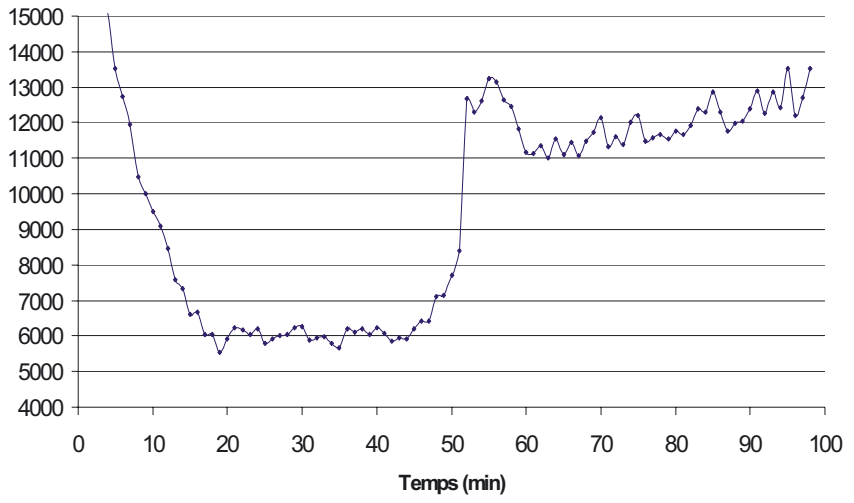

Fig. 6. Évolution du nombre de particule de $0,3 \mu \mathrm{m}$ en fonction du temps (Linoléum antidérapant).

particules par litre dans la cellule test jusqu'à atteindre 2000 part./1 pour le linoléum lisse et 6000 part./1 pour le linoléum rugueux.

Cette différence est due au fait que le générateur d'aérosol utilisé (TOPAS) ne permet pas de spécifier la quantité de particules à injecter, la comparaison entre les deux revêtement sera faite en se basant sur la différence entre les deux paliers de pollution.

On remarque sur les graphiques que l'ampleur de la remise en suspension est sensiblement la même pour les deux revêtements : de 2000 particule/litre à 5000 particule/litre pour le linoléum lisse, et de 6000 à approximativement 12000 particule/litre pour le linoléum rugueux (du simple au double pratiquement).

La seule différence notable est la durée de cette remise en suspension : 20 min pour le revêtement lisse contre 10 min pour le revêtement rugueux. Ceci peut s'expliquer par le fait que aspérités présentes sur la surface rugueuse de second revêtement provoque une friction plus importante avec les semelles des chaussures, contribuant ainsi à l'accélération de la remise en suspension des particules déposées.

\section{Conclusion et perspectives}

La prédiction de la qualité particulaire de l'air des ambiances intérieures est très complexe. En effet, l'évaluation des niveaux de pollution particulaire à l'intérieur des bâtiment nécessite la connaissance de deux entités en interaction : l'air chargé de particules solides et l'ambiance intérieure du bâtiment.

La remise en suspension des particules par les pas humains est un phénomène encore peu connu [5]. La quantité de paramètres entrant en jeu est considérable : électricité statique, vibrations, débit de VMC, nature de polluant...

Une réflexion théorique et une approche numérique (modélisation de la remise en suspension par les pas) sont en cours. Celles-ci se basent sur l'établissement de corrélations entre l'ampleur de la remise en suspension et la nature du revêtement, le nombre des occupants, leurs corpulences, le type de chaussures, l'intensité de l'activité, etc., afin d'intégrer dans un code de prédiction, le terme source exprimé précédemment dans l'équation du bilan.

En effet, les pas de marche peuvent être assimilés à une injection de particules, ponctuelle et discontinue dans l'espace et dans le temps.

\section{Références}

[1] A.C.K. Lai, F. Chen, Modeling particle deposition and distribution in a chamber with a two-equation Reynoldsaveraged Navier-Stokes model, Aerosol Science 37 (2006) $1770-1780$

[2] W.W. Nazaroff, G.R. Cass, Particle deposition from a natural convection flow onto a vertical isothermal flat plate, J. Aerosol Sci. 20 (1989) 138-139

[3] M. Abadie, Contribution à l'étude de la pollution particulaire : rôle des parois, rôle de la ventilation, Thèse Université de La Rochelle, Leptab, 2000

[4] J. Bouilly, Étude de l'Impact de la pollution particulaire sur la qualité de l'air intérieur en site urbain, Thèse Université de La Rochelle, Leptab, 2003

[5] C.A.S. Gomes, B. Hu, J.D. Freihaut, Resuspension of allergen-containig particles under mechanical and aerodynamic forces from human walking. Intrudoction to an experimental methodology, Indoor Environment Center, Architectural department, University Park, PA 16802, USA (2006)

[6] K. Limam, et al., Programme Interministériel pour une Meilleure Qualité de l'air à l'échelle locale et urbaine. Caractérisation physico-chimique et étude du transport des particules à l'intérieur des locaux, Projet National PRIMEQUAL2-MEDD- 2002/2007

[7] Rapport AK0609-LEPTAB-2, Qualification comparative de dureté des revêtements, septembre 2006 